\title{
The hypocretin/orexin receptor-1 as a novel target to modulate cannabinoid
}

reward

África Flores, Rafael Maldonado and Fernando Berrendero ${ }^{\#}$

Laboratory of Neuropharmacology, Department of Experimental and Health Sciences, Universitat Pompeu Fabra, PRBB, C/ Doctor Aiguader 88, 08003 Barcelona, Spain

${ }^{\#}$ Corresponding author: Fernando Berrendero, Phone: +34-93-3160890; Fax: + 34-93-

3160901; E-mail: fernando.berrendero@upf.edu

Keywords: WIN55,212-2, hypocretin, cannabinoid, reward, dopamine, knockout

Number of words (abstract): 250

Number of words (text): 3982

Number of figures: 5

Number of tables: 1

Supplementary material: 1 (Materials and Methods and Supplementary Table 1) 
Background: Cannabis is the most widely used illicit drug in the world. Although there is a high prevalence of users who seek treatment for cannabis dependence, no accepted pharmacological treatment is still available to facilitate and maintain abstinence. The hypocretin/orexin system plays a critical role in drug addiction and the potential participation of this system in the addictive properties of cannabinoids is still unknown. Methods: We investigated the effects of hypocretins in the intravenous selfadministration of the synthetic cannabinoid agonist WIN55,212-2 using hypocretin receptor-1 (Hcrtr-1) and hypocretin receptor-2 (Hcrtr-2) antagonists, and Hcrtr-1 knockout mice. Additional groups of mice were trained to obtain water to rule out operant responding impairments. Activation of hypocretin neurons was analyzed by using double label immunofluorescence of FosB/ $\Delta$ FosB with hypocretin-1. Microdialysis studies were performed to evaluate dopamine extracellular levels in the nucleus accumbens after acute $\Delta^{9}$-tetrahydrocannabinol (THC) administration.

Results: Systemic administration of the Hcrtr-1 antagonist SB334867, but not the Hctr2 antagonist TCSOX229, reduced intravenous self-administration of WIN55,212-2 as well as the maximum effort to obtain a WIN55,212-2 infusion as revealed under a progressive ratio schedule. This role of Hcrtr-1 in the reinforcing and motivational properties of WIN55,212-2 was confirmed in Hcrtr-1 knockout mice. Contingent, but not non-contingent WIN55,212-2 self-administration increased the percentage of hypocretin cells expressing FosB/ $\Delta$ FosB in the lateral hypothalamus. The enhancement in dopamine extracellular levels in the nucleus accumbens induced by THC was blocked in mice lacking the Hcrtr-1.

Conclusions: These findings demonstrate that Hcrtr-1 modulates the reinforcing properties of cannabinoids, which could have a clear therapeutic interest. 


\section{Introduction}

Cannabis is the most used illicit drug worldwide (1). It is estimated that about $9 \%$ of those who ever use this drug become daily users (2), rising to $16 \%$ when the consumption is initiated during the adolescence (2). Recent analysis of cannabis extracts has shown an increase in potency over the last years due to enhanced content of $\Delta^{9}$ tetrahydrocannabinol (THC), its primary psychoactive constituent (3). A high THC content leads to an increase in the subjective effects of this drug probably enhancing the risk of dependence and psychotic symptoms of cannabis users (3). Treatment admissions for cannabis-use disorders have progressively increased in the past decade. Approximately $90 \%$ of those seeking treatment for these disorders have great difficulty achieving and sustaining periods of abstinence (4), and the majority relapse to use following therapeutic interventions (5). There are currently no effective pharmacotherapeutic approaches for this disorder (5). Therefore, the need for identifying specific medications to improve treatment outcomes for cannabis dependence becomes a major clinical priority.

Several findings suggest that the hypocretin/orexin system could be a potential target for the development of new medications for cannabis abuse. Hypocretin-1 and -2 (also known as orexin A and B) are lateral hypothalamic neuropeptides that project throughout the brain (6). Two G-protein coupled receptors that respond to hypocretins have been characterized, hypocretin receptor-1 (Hcrtr-1) and hypocretin receptor-2 (Hcrtr-2) (7). The hypocretin system has been reported to play an important role in the reward circuits and to participate in the reinforcing properties of psychostimulants, nicotine, opioids and alcohol $(8,9)$. Several reports have described the existence of functional interactions between the hypocretin and the endocannabinoid systems, mainly in the regulation of appetite and analgesia (10). However, whether hypocretins 
take part of the neurobiological substrate underlying the addictive properties of cannabinoids remains to be elucidated.

In this study, we investigated the involvement of the hypocretin system in the modulation of the reinforcing effects of cannabinoids and their ability to elevate extracellular dopamine levels in the nucleus accumbens (NAc). For this purpose, we used specific Hcrtr-1 and Hcrtr-2 antagonists, as well as Hcrtr-1 knockout (KO) mice. 


\section{Materials and methods}

\section{Animals}

Experiments were performed in male C57BL/6J mice (Charles River, L'Arbresle, France) and in male Hcrtr-1 KO and their wild-type (WT) littermates (B6.129P2Hcrtr1tm1Dgen/J, Jackson Laboratories) (10-16 week old). KO mice were crossed at least 7 generations to $\mathrm{C} 57 \mathrm{BL} / 6 \mathrm{~J}$ mice. Hcrtr-1 $\mathrm{KO}$ mice were genotyped using the following primers: 5'-TCTCACTGAGCATGCTCCCTGGGTG-3', 5'GGGCCAGCTCATTCCTCCCACTCAT-3' ${ }^{\prime}$ and 5'- $^{\prime}$ AGAGTGTTCGGGATGTTCCGCCAAG-3'. The multiplex PCR produces a 379-bp band for the WT allele and a 538-bp band for the KO allele and both for heterozygous mice. Mice were housed one per cage in a temperature- $\left(21 \pm 1^{\circ} \mathrm{C}\right)$ and humidity$(55 \pm 10 \%)$ controlled room with a 12-h light/dark cycle (light between 08:00 to 20:00) for in vivo microdialysis study. For self-administration studies, mice were single housed and exposed to a reversed light/dark cycle conditions (lights off 8:00 A.M.) and the experiments took place during the dark phase. Indeed, previous studies show that hypocretin $\mathrm{KO}$ mice do not acquire an operant behavior maintained by food or water reward during the light phase, but their operant responses are not modified relative to WT mice when the KO mice are trained for these reward stimuli during the dark phase (11). Mice were habituated to the reversed cycle conditions for 1 week before starting the training sessions. All animal procedures were conducted in accordance with the guidelines of the European Communities Directive 86/609/EEC regulating animal research and approved by the local ethical committee (CEEA-IMAS-UPF), and the statement of compliance with standards for use of laboratory animals by foreign institutions nr. A5388-01 approved by the National Institutes of Health (USA). 


\section{Drugs}

The cannabinoid agonist inducing the most reliable operant intravenous selfadministration in our experimental conditions, WIN55,212-2 (12) was used. WIN55,212-2 (Sigma) was dissolved in one drop of Tween 80 and diluted in physiological saline $(0.9 \% \mathrm{NaCl})$. For the self-administration sessions, WIN55,212-2 was contingently administered by intravenous (iv) route at $12.5 \mu \mathrm{g} / \mathrm{kg}$ per infusion. The cannabinoid agonist producing the most reliable enhancement of dopamine extracellular levels in the NAc in our experimental conditions, THC (13) was used for in vivo microdialysis studies. THC $(0.3 \mathrm{mg} / \mathrm{kg})$ (THC-Pharm-GmbH) was dissolved in $5 \%$ ethanol and 5\% cremophor in physiological saline and administered by intraperitoneal (ip) route in a volume of $10 \mathrm{ml} / \mathrm{kg}$ body weight. The Hcrtr-1 antagonist SB334867 $(10 \mathrm{mg} / \mathrm{kg})$ (Tocris) was dissolved in 1\% (2-hydroxypropyl)- $\beta$-cyclodextrin (Sigma) and 10\% DMSO in distilled water. The Hcrtr-2 antagonist TCSOX229 (10mg/kg) (Tocris) was dissolved in physiological saline. Both hypocretin antagonists were administered by ip route in a volume of $5 \mathrm{ml} / \mathrm{kg}$ body weight. The SB334867 and TCSOX229 doses were based on previous studies and did not modify locomotor activity $(14,15)$.

\section{WIN55,212-2 self-administration}

Apparatus. The experiments were conducted in mouse operant chambers (model ENV-307A-CT; Med Associates Inc.) equipped with two holes, one randomly selected as the active hole and the other as the inactive hole, as previously described (16).

Jugular vein catheterization. Mice were anesthetized with a ketamine/xylazine mixture (ip route) and then implanted with indwelling intravenous silastic catheters on their right jugular vein, as previously described (16). After surgery, animals were 
individually housed and allowed to recover for 4 days before initiation of selfadministration sessions.

Drug self-administration training. Slight modifications were done to the operant model previously described (12). See Supplement 1 for further details.

Genetic (Hcrtr-1 KO mice) and pharmacological (SB334867 and TCSOX229 administration) approaches were used in these experiments. For the pharmacological studies, mice received an injection of SB334867 or TCSOX229 $(10 \mathrm{mg} / \mathrm{kg}$, ip) 30 minutes before each daily session of WIN55,212-2 self-administration. In another set of experiments, SB334867 or TCSOX229 were acutely administered on day 9 after stable acquisition of WIN55,212-2 self-administration.

\section{Water-maintained operant behavior}

To exclude nonspecific effects on learning or memory due to the SB334867 or TCSOX229 pretreatments or Hcrtr-1 gene deletion, we evaluated operant responding maintained by water delivery in water-deprived mice, as previously reported (16). Water-maintained operant behavior has been widely used previously to discard nonspecific effects on learning and memory under similar pharmacological and genetic conditions $(17,18,19)$. See Supplement 1 for further details.

\section{Immunofluorescence studies}

See Supplement 1.

\section{In vivo microdialysis}

The evaluation of the extracellular dopamine levels in the NAc was performed as previously reported (13). See Supplement 1 for further details. 


\section{Statistical analysis}

Differences in WIN55,212-2 self-administration between pretreatments/genotypes were analyzed using a three-way analysis of variance (ANOVA) with hole and pretreatment/genotype as between-subject factors, and time as within-subject factor. Water-maintained operant responding was analyzed similarly. Pearson's $\chi^{2}$ test was used to compare the percentage of acquisition between pre-treatments/genotypes. Differences in the breaking points were analyzed using two tailed unpaired Student ttests. Immunofluorescence studies were analyzed by one-way ANOVA. The microdialysis data were analyzed using three-way ANOVA with treatment and genotype as between-subject factors, and time after THC injection as within-subject factor. AUC values were analyzed by two-way ANOVA with genotype and treatment as between-subject factors. When appropriate, post-hoc individual comparisons were determined using the Bonferroni test. The level of significance was $p<0.05$ in all experiments. 


\section{Results}

Hypocretin receptor-1 blockade reduces acquisition of WIN55,212-2 selfadministration behavior

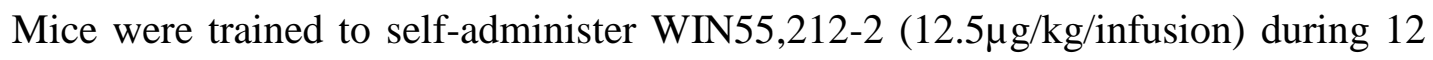
consecutive days and received an ip injection of vehicle, the Hcrtr-1 antagonist SB334867 $(10 \mathrm{mg} / \mathrm{kg})$ or the Hcrtr-2 antagonist TCSOX229 $(10 \mathrm{mg} / \mathrm{kg}) 30$ minutes before each self-administration session. SB334867 reduced the percentage of acquisition of WIN55,212-2 self-administration (Figure 1A). Thus, 75\% of mice receiving vehicle achieved the acquisition criteria, whereas only $29 \%$ of SB334867 pretreated mice acquired the behavior $\left(\chi^{2}=6.46, \mathrm{p}<0.01\right)$. Three-way ANOVA with repeated measures revealed a significant interaction between hole and day $\left(\mathrm{F}_{(11,308)}=4.42, \mathrm{p}<0.01\right)$, indicating a progress in operant responding for WIN55,212-2 across days (Figure 1B) (see Table 1 for three-way ANOVA). Active and inactive nosepoking expressed as the area under the curve (AUC) values were analyzed during the period where the animals showed a stable behavior of self-administration (days 7-12). Two-way ANOVA revealed an interaction between hole and pretreatment $\left(\mathrm{F}_{(1,28)}=4.19\right.$, $\mathrm{p}<0.05)$. Subsequent one-way ANOVA showed a clear trend to decrease the active responses in mice treated with the Hcrtr-1 antagonist ( $\mathrm{p}=0.07$ ) (Figure 1C). Conversely, acquisition of WIN55,212-2 self-administration was not affected by TCSOX229 pretreatment. Thus, $77 \%$ of mice pretreated with vehicle and $90 \%$ of mice pretreated with TCSOX229 acquired this behavior $\left(\chi^{2}=0.67\right.$, NS) (Figure 1D). Moreover, pretreatment with TCSOX229 did not modify the active responses from day 7 to 12 as shown by AUC values (Figure 1E and 1F) (see Table 1 for three-way ANOVA).

To exclude unspecific effects on learning or memory due to the treatment with SB334867 and TCSOX229, we studied the effects of these antagonists in operant 
responding maintained by water delivery in water-deprived mice. SB334867 or TCSOX229 pretreatments did not produce any effect on this operant performance (Figure 1G and 1I). Thus, all the mice pretreated with SB334867, TCSOX229 or respective vehicles acquired the operant behavior. Moreover, the number of active responses during the whole training and the breaking point in the PR schedule of reinforcement were similar in all groups of mice (Figure $1 \mathrm{H}$ and $1 \mathrm{~J}$ ).

\section{Acute administration of the Hcrtr-1 antagonist SB334867 reduces WIN55,212-2 self-administration and motivation to obtain the drug}

We next evaluated the effects of Hcrtr-1 and Hcrtr-2 blockade on WIN55,212-2 self-administration after stable acquisition of this behavioral response. All mice were pretreated with vehicle 30 minutes before each session of WIN55,212-2 selfadministration from day 1 to 8 . Those mice not achieving the acquisition criteria by day $8(22 \%)$ were discarded. On day 9, mice received an acute injection of SB334867 $(10 \mathrm{mg} / \mathrm{kg})$ or TCSOX229 $(10 \mathrm{mg} / \mathrm{kg})$ instead of vehicle, and from day 10 to 12 day mice were pretreated again with vehicle. Acute SB334867 injection reduced WIN55,212-2 self-administration behavior (Figure 2A). Thus, two-way ANOVA with repeated measures showed a significant interaction between hole and treatment $\left(F_{(2,30)}=20.55\right.$, $\mathrm{p}<0.001)$. Subsequent post hoc analysis revealed a reduction of active responses on day $9(6.2 \pm 1.3)$ when compared with the mean responses of the three days before $(20.7 \pm 1.5$, $\mathrm{p}<0.01)$ and the three days after $(16.2 \pm 1.3, \mathrm{p}<0.01)$. No differences were observed in active nose-poking between the three days before and after SB334867 treatment, indicating a recovery of WIN55,212-2 self-administration behavior. Importantly, no difference was revealed for inactive nose-poking, suggesting that this effect was not caused by nonspecific consequences on locomotion. In contrast, acute TCSOX229 
injection on day 9 did not modify WIN55,212-2 self-administration (Figure 2B). Thus, two-way ANOVA with repeated measures revealed no interaction between hole and treatment $\left(\mathrm{F}_{(2,22)}=1.48\right.$, NS), suggesting that Hcrtr-1 plays a specific role in the modulation of this behavior.

To evaluate the maximum effort to obtain a WIN55,212-2 infusion, an additional group of mice was trained to self-administer WIN55,212-2 under FR1 during 12 days and then moved to a PR schedule of reinforcement on day 13. Mice were pretreated with SB334867, TCSOX229 or respective vehicles 30 minutes before the PR session. As shown in Figure 2C and 2D, acute SB334867, but not TCSOX229 administration reduced the breaking point when compared to the control group $(\mathrm{p}<0.01)$. These results suggest that Hcrtr-1, but not Hcrtr-2 participates in the motivation to obtain WIN55,212-2.

\section{Hypocretin receptor-1 gene deletion impairs WIN55,212-2 self-administration and motivation to obtain the drug}

The participation of Hcrtr-1 in the reinforcing properties of WIN55,212-2 was further evaluated by using Hcrtr-1 KO mice and their WT littermates. A decrease in the percentage of acquisition of WIN55,212-2 self-administration was observed in mutant animals (Figure 3A). Thus, the percentage of mice that achieved the acquisition criteria for WIN55,212-2 self-administration was $75 \%$ for WT and 33\% for KO mice $\left(\chi^{2}=5.90\right.$, $\mathrm{p}<0.01)$. Moreover, mutant mice displayed a lower number of active responses than WT animals (Figure 3B). Thus, three-way ANOVA with repeated measures revealed a significant interaction between hole, day, and genotype $\left(\mathrm{F}_{(11,352)}=2.41, \mathrm{p}<0.01\right)$. Subsequent post hoc analysis for each day of training revealed a significant decrease in active responding in Hcrtr-1 KO when compared with WT mice from days 8 to 12 
(Figure 3B). Accordingly, two-way ANOVA calculated for AUC values (days 7-12) showed an interaction between hole and genotype $\left(\mathrm{F}_{(1,32)}=5.05, \mathrm{p}<0.05\right)$ (Figure 3C). Subsequent post hoc analysis revealed that the active responses displayed by WT animals were higher than in mutants $(\mathrm{p}<0.01)$. The maximum effort to obtain a WIN55,212-2 infusion was evaluated under a PR schedule one day after the last selfadministration session. The breaking point values for Hcrtr-1 $\mathrm{KO}$ mice were significantly lower when compared with wild-type animals $(\mathrm{p}<0.05)$ (Figure 3D). In agreement with the pharmacological experiments, these data reveal an impairment in the performance of operant WIN55,212-2 self-administration behavior in mice lacking the Hcrtr-1.

To rule out unspecific effects on learning or memory due to the Hcrtr-1 gene deletion, KO and WT mice underwent an operant conditioning maintained by water. The lack of Hcrtr-1 did not produce any effect on the acquisition of this behavior, the number of responses during the whole training (Figure 3E), or the breaking point values in PR schedule of reinforcement (Figure 3F).

\section{Contingent, but not non-contingent WIN55,212-2 self-administration activates hypocretin neurons in the lateral hypothalamus}

To further characterize the role of hypocretin neurons in cannabinoid reinforcing effects, we studied the possible activation of these cells during WIN55,212-2 selfadministration using double label immunofluorescence of FosB/ $\Delta$ fosB with hypocretin1 in the lateral hypothalamus (LH), and the dorsomedial and perifornical hypothalamus (DMH/PFA). In order to distinguish between the pharmacological responses of WIN55,212-2 and the effects related to drug-seeking, a contingent/non-contingent WIN55,212-2 self-administration paradigm was performed during 12 consecutive days 
(Figure 4A, 4B and 4C). Similarly to the previous experiments, $70 \%$ of the contingent mice met all the acquisition criteria within $6.0 \pm 0.7$ days, and showed a mean cumulative intake of $5.0 \pm 0.7 \mathrm{mg} / \mathrm{kg}$ of WIN55,212-2 during the entire training period (Figure 4C). Saline- and WIN-yoked animals did not discriminate between holes during the training sessions (Figure 4A and 4B). Contingent WIN55,212-2 self-administration increased the percentage of hypocretin cells expressing FosB/AFosB in the LH (Figure 4D and 4F). Thus, one-way ANOVA revealed a significant effect of treatment $\left(\mathrm{F}_{(2,18)}=6.13\right.$, $\mathrm{p}<0.01$ ), and subsequent post hoc analysis showed an activation of hypocretin neurons in mice receiving WIN55,212-2 contingently, but not in those receiving saline or WIN55,212-2 passively $(\mathrm{p}<0.05)$ (Figure $4 \mathrm{D}$ and $4 \mathrm{~F})$. These data suggest that hypocretin neurons in the LH are activated by operant seeking of WIN55,212-2 reinforcing effects. In contrast, the contingent self-administration of WIN55,212-2 did not activate the hypocretinergic neurons in the DMH/PFA when compared to salineyoked mice (Figure 4E), although a reduction of FosB/ $\Delta$ FosB expression in hypocretin neurons was observed in animals receiving WIN55,212-2 passively when compared with those receiving WIN55,212-2 contingently $(\mathrm{p}<0.05)$.

To ensure that activation of hypocretin neurons in the LH was not a consequence of the learning process involved in the acquisition of an operant behavior, a contingent/non-contingent operant conditioning maintained by water was performed in water-deprived mice (Figure $4 \mathrm{H}$ and $4 \mathrm{I}$ ). All the mice administering water contingently met the acquisition criteria while animals receiving water passively did not discriminate between holes during the training period. Double immunofluorescence of FosB/ $\Delta$ FosB with hypocretin-1 revealed that this operant behavior did not induce the activation of hypocretin cells located in the $\mathrm{LH}$, although an increase of FosB/ $\Delta$ FosB expression was observed in hypocretin neurons in the DMH/PFA (Figure 4J and 4K). The percentage of 
hypocretin cells expressing FosB/ $\Delta$ FosB was higher in water-yoked animals than in saline-yoked mice in both the LH and the DMH/PFA may be due to the stress induced by the water deprivation $(20,21)$. A table containing the total number of hypocretin, FosB/ $\Delta$ FosB neurons and hypocretin/FosB/ $\Delta$ FosB double positive cells of each experimental condition in Figure 4 is shown (Supplementary Table 1).

\section{The enhanced of dopamine extracellular levels in the nucleus accumbens induced by THC is blocked in hypocretin receptor-1 knockout mice}

Given the role of the mesolimbic dopamine system in cannabinoid reinforcing properties (22), we investigated the effects of the deletion of Hcrtr-1 in the enhancement of dopamine extracellular levels in the NAc induced by THC $(0.3 \mathrm{mg} / \mathrm{kg}$, ip $)$ by in vivo microdialysis. This was the only dose of THC that produced a reliable enhancement of dopamine levels in the NAc under our experimental conditions (13). Three-way ANOVA showed a significant interaction between genotype and treatment $\left(\mathrm{F}_{(1,44)}=4.83\right.$, $\mathrm{p}<0.05)$ without other two- or three-way interactions (Figure 5C). THC increased dopamine extracellular levels in the NAc in WT mice, and this effect was not observed in Hcrtr-1 KO mice, as shown by AUC values (Figure 5D). Thus, two-way ANOVA revealed a significant interaction between genotype and treatment $\left(\mathrm{F}_{(1,44)}=4.77, \mathrm{p}<0.05\right)$, and post hoc analysis showed a significant effect of THC in WT $(p<0.01)$ but not in KO animals, as well as significant differences between WT and mutant mice when receiving THC $(\mathrm{p}<0.01)$. These results indicate that the deletion of the Hcrtr-1 impairs the effects of THC on the extracellular levels of dopamine in the NAc. 


\section{Discussion}

This study demonstrates that Hcrtr-1, but not Hcrtr-2 modulates the operant selfadministration of WIN55,212-2 and the motivation to obtain this drug. In agreement, contingent self-administration, but not passive administration of WIN55,212 activates hypocretin neurons of the LH. The capability of THC to increase dopamine levels in the NAc is blocked in Hcrtr-1 KO mice suggesting that hypocretins modulate cannabinoid reinforcing properties through a dopamine-dependent mechanism. Together, these data reveal the interest of Hcrtr-1 for the development of novel treatments for cannabis abuse in humans.

Chronic treatment with the Hcrtr-1 antagonist SB334867 impaired WIN55,212-2 self-administration since only $29 \%$ of mice receiving this treatment acquired the acquisition criteria in comparison with $75 \%$ of control animals. In agreement, the acute SB334867 administration after the acquisition of WIN55,212-2 self-administration blocked this behavioral response, as demonstrated by the decrease in responding on the active hole and the lack of change on inactive nose-poking. Moreover, SB334867 injection reduced the maximal effort required to obtain a WIN55,212-2 infusion in a PR schedule. The impairment of WIN55,212-2 self-administration cannot be attributed to possible learning deficits in the acquisition of an operant behavior since SB334867 did not alter operant responding for a natural reward, such as water. Consistent with our results, Hcrtr-1 signaling has also been reported to modulate the reinforcing effects of opioids $(23,24)$, nicotine $(25)$ and alcohol $(26,27)$. However, the involvement of the hypocretin system in the rewarding properties of cocaine has mainly been revealed under conditions that require greater degrees of effort and motivation to obtain the drug $(28,29)$. The present data point to a specific role for Hcrtr-1 in the modulation of the reinforcing properties of cannabinoids since the Hcrtr-2 antagonist TCSOX229 did not 
alter this behavior. Although both hypocretin receptor subtypes are localized in brain areas important for reward processing such as the ventral tegmental area (VTA), NAc and bed nucleus of the stria terminalis $(30,31)$, there is little evidence about a possible role for Hcrtr-2 in the regulation of the rewarding properties of drugs of abuse.

Consistent with these pharmacological results, the operant WIN55,212-2 selfadministration was impaired in Hcrtr-1 KO mice. Thus, $75 \%$ of WT animals acquired the acquisition criteria in comparison with $33 \%$ of mutant mice. In addition, the active responses and the maximal effort required to obtain a drug infusion were reduced in mice lacking the Hcrtr-1. Importantly, these mice did not show any deficit in the performance of an operant conditioning maintained by water, suggesting that the decreased responding for WIN55,212-2 was not secondary to disruption in learning processes. In agreement, it has been previously shown that these $\mathrm{KO}$ mice maintain normal operant responding for food reinforcers (32). Taken together, our pharmacological and genetic findings provide definitive evidence that Hcrtr-1 is essential for the reinforcing properties of WIN55,212-2.

Operant yoked-control models of drug self-administration are essential to understand whether neurochemical changes are attributable to the effects of drugseeking or to the pharmacological actions of the drug $(33,34)$. In agreement with the behavioral results, we found that contingent self-administration of WIN55,212-2 induced an increase in the percentage of hypocretin neurons expressing FosB/ $\Delta F$ os B in the LH. Notably, hypocretin cells were not activated in mice receiving passive infusions of the cannabinoid, suggesting that the recruitment of hypocretin cells within the LH is mainly due to operant seeking for the reinforcing effects of this drug. Moreover, operant conditioning maintained by water did not modify FosB/AFosB expression in LH hypocretin cells in comparison with water-yoked mice, indicating that these neurons are 
not recruited due to the learning process involved in the acquisition of an operant behavior. However, the percentage of hypocretin cells expressing FosB/ $\Delta$ FosB was higher in water-yoked animals than in saline-yoked mice in this brain area. The contingent/non-contingent operant conditioning maintained by water was performed in water-deprived mice, and the stress induced by this mandatory water restriction $(20,21)$ could participate in the high basal activation observed in this brain area. On the other hand, WIN55,212-2 contingent self-administration did not affect the hypocretin neurons in the DMH/PFA, although a decrease of FosB/ $\Delta$ FosB expression in these cells was observed in mice receiving WIN55,212-2 passively versus those receiving the drug contingently. This effect could be related to the learning process because DMH/PFA hypocretin cells were activated during an operant conditioning maintained by water. The existence of a functional dichotomy for hypocretin neurons has been proposed, with hypocretin cells located in the LH mainly involved in reward processing while those located in the DMH/PFA playing a role in the regulation of arousal and stress (35). Thus, Fos expression in LH hypocretin cells, and not those in DMH/PFA was correlated with morphine and cocaine conditioned place preference (23). A correlation between morphine place preference and Fos expression in LH hypocretin cells projecting to the rostral part of the VTA has also been recently shown (36). In agreement, chronic ethanol drinking increased mRNA hypocretin expression in the $\mathrm{LH}$, but not in the DMH/PFA (26), although another study observed this effect only after acute ethanol administration (37). On the contrary, an activation of hypocretin neurons located in the LH has been reported during the dysphoric state associated to nicotine (15) and morphine (38) withdrawal. Therefore, further studies are required to support the possible different physiological role of the diverse hypocretin cell populations. Indeed, WIN55,212-2 reinforcing effects were associated to a selective activation of LH 
hypocretin neurons in the present study, but high basal activation of these cells were observed after water deprivation probably due to the stress state induced by this experimental procedure.

The stimulation of dopamine mesolimbic neurotransmission is essential for the reinforcing effects of many drugs of abuse, including cannabinoids $(39,40)$. In the VTA, $\mathrm{CB}_{1}$ cannabinoid receptors are located on presynaptic glutamatergic and GABAergic neurons where they modulate the excitatory and inhibitory inputs on mesolimbic dopaminergic neurons (41). According to our behavioral results, systemic injection of THC increased extracellular dopamine levels in the NAc in WT animals, but this effect was blocked in Hcrtr-1 KO mice. VTA hypocretin signaling seems to be particularly important in the regulation of drug reward seeking behavior (42). Thus, the VTA shows high density of hcrtr-1 $(28,43)$, hypocretin-1 induces a direct depolarization of VTA dopamine neurons (44), and the intra-VTA infusion of hypocretin-1 increases dopamine levels in the NAc $(43,45)$. Although the exact neuronal mechanism is unclear at present, our findings suggest that cannabinoid exposure could induce the release of hypocretins in the VTA following the activation of these neurons in the LH, which directly project to the VTA (46). The subsequent activation of hcrtr-1 by hypocretins in the VTA would be essential for the rewarding properties of cannabinoids through the modulation of the dopamine mesolimbic pathway. In agreement with a functional interaction between hypocretins and cannabinoids in reward-related processes, it has been recently shown that the intra-VTA administration of the $\mathrm{CB}_{1}$ cannabinoid antagonist AM251 blocks the conditioned place preference induced by the chemical stimulation of LH hypocretin neurons (47). Moreover, hcrtr-1 and $\mathrm{CB}_{1}$ cannabinoid receptors form heterodimers (48) suggesting the existence of a cross-talk between these two systems which will require future investigation. 
In conclusion, our findings show that hypocretin transmission at Hcrtr-1 is a critical component involved in the reinforcing and motivational properties of cannabinoids. Therefore, Hcrtr-1 antagonists could represent an interesting pharmacological tool for cannabis dependence in humans which is a major clinical need devoid of available treatment at the present moment. 


\section{Acknowledgments}

This work was supported by the Instituto de Salud Carlos III grants, \#PI07/0559, \#PI10/00316 and \#RD06/001/001 (RTA-RETICS), by the Spanish Ministry of Science and Technology, Consolider-C \#SAF2007-64062 and \#SAF2011-29864, the Catalan Government (SGR2009-00731), and by the Catalan Institution for Research and Advanced Studies (ICREA Academia program). África Flores is recipient of a predoctoral fellowship from the Spanish Ministry of Education. We thank Dulce Real and Marta Linares for invaluable technical assistance.

\section{Financial Disclosures}

The authors have no conflicts of interest to declare. 


\section{References}

1. SAMHSA, Substance Abuse and Mental Health Services Administration (2010). Results from the 2009 National Survey on Drug Use and Health: Volume I. Summary of National Findings

2. Anthony JC. The epidemiology of cannabis dependence. In: Roffman RA, Stephens RS, eds. Cannabis dependence: its nature, consequences and treatment. Cambridge, UK: Cambridge University Press, 2006: 58-105.

3. Hall W, Degenhardt L (2009): Adverse health effects of non-medical cannabis use. Lancet 374:1383-1391.

4. Budney AJ, Hughes JR (2006): The cannabis withdrawal syndrome. Curr Opin Psychiatry 19:233-238.

5. Vandrey R, Haney M (2009): Pharmacotherapy for cannabis dependence: how close are we? CNS Drugs 23:543-553.

6. Peyron C, Tighe DK, van den Pol AN, de Lecea L, Heller HC, Sutcliffe JG, et al. (1998): Neurons containing hypocretin (orexin) project to multiple neuronal systems. J Neurosci 18: 9996-10015.

7. Sakurai T, Amemiya A, Ishii M, Matsuzaki I, Chemelli RM, Tanaka H, et al. (1998): Orexins and orexin receptors: a family of hypothalamic neuropeptides and $\mathrm{G}$ protein-coupled receptors that regulate feeding behavior. Cell 92: 573585.

8. Aston-Jones G, Smith RJ, Sartor GC, Moorman DE, Massi L, Tahsili-Fahadan P, et al. (2010): Lateral hypothalamic orexin/hypocretin neurons: A role in reward-seeking and addiction. Brain Res 1314:74-90. 
9. Plaza-Zabala A, Maldonado R, Berrendero F (2012): The hypocretin/orexin system: implications for drug reward and relapse. Mol Neurobiol 45:424-439.

10. Kukkonen JP (2012): Physiology of the orexinergic/hypocretinergic system: a revisit in 2012. Am J Physiol Cell Physiol 304:C2-C32.

11. McGregor R, Wu MF, Barber G, Ramanathan L, Siegel JM (2011). Highly specific role of hypocretin (orexin) neurons: differential activation as a function of diurnal phase, operant reinforcement versus operant avoidance and light level. J Neurosci 31:15455-15467.

12. Mendizábal V, Zimmer A, Maldonado R (2006): Involvement of kappa/dynorphin system in WIN55,212-2 self-administration in mice. Neuropsychopharmacology 31:1957-1966.

13. Robledo P, Trigo JM, Panayi F, de la Torre R, Maldonado R (2007): Behavioural and neurochemical effects of combined MDMA and THC administration in mice. Psychopharmacology (Berl) 195:255-264.

14. Plaza-Zabala A, Martín-García E, de Lecea L, Maldonado R, Berrendero F (2010): Hypocretins regulate the anxiogenic-like effects of nicotine and induce reinstatement of nicotine-seeking behavior. J Neurosci 30:2300-2310.

15. Plaza-Zabala A, Flores Á, Maldonado R, Berrendero F (2012): Hypocretin/orexin signaling in the hypothalamic paraventricular nucleus is essential for the expression of nicotine withdrawal. Biol Psychiatry 71:214-223.

16. Soria G, Mendizábal V, Touriño C, Robledo P, Ledent C, Parmentier M, et al. (2005) Lack of CB1 cannabinoid receptor impairs cocaine self-administration. Neuropsychopharmacology 30:1670-1680. 
17. Trigo JM, Renoir T, Lanfumey L, Hamon M, Lesch KP, Robledo P, et al. (2007). 3,4-methylenedioxymethamphetamine self-administration is abolished in serotonin transporter knockout mice. Biol Psychiatry 62:669-679.

18. Roth-Deri I, Mayan R, Yadid G (2006). A hypothalamic endorphinic lesion attenuates acquisition of cocaine self-administration in the rat. Eur Neuropsychopharmacol 16:25-32.

19.Ward SJ, Rosenberg M, Dykstra LA, Walker EA (2009). The CB1 antagonist rimonabant (SR141716) blocks cue-induced reinstatement of cocaine seeking and other context and extinction phenomena predictive of relapse. Drug Alcohol Depend 105:248-255.

20. Csaba G, Kovács P, Tóthfalusi L, Pállinger E (2005). Prolonged effect of stress (water and food deprivation) at weaning or in adult age on the triiodothyronine and histamine content of immune cells. Horm Metab Res 37:711-715.

21.Roberts EM, Pope GR, Newson MJ, Lolait SJ, O'Carroll AM (2011). The vasopressin $\mathrm{V} 1 \mathrm{~b}$ receptor modulates plasma corticosterone responses to dehydration-induced stress. J Neuroendocrinol 23:12-19.

22. Solinas M, Scherma M, Fattore L, Stroik J, Wertheim C, Tanda G, et al. (2007): Nicotinic alpha 7 receptors as a new target for treatment of cannabis abuse. $J$ Neurosci 27:5615-5620.

23. Harris GC, Wimmer M, Aston-Jones G (2005): A role for lateral hypothalamic orexin neurons in reward seeking. Nature 437:556-559.

24. Smith RJ, Aston-Jones G (2012): Orexin/hypocretin 1 receptor antagonist reduces heroin self-administration and cue-induced heroin seeking. Eur $J$ Neurosci 35:798-804. 
25. Hollander JA, Lu Q, Cameron MD, Kamenecka TM, Kenny PJ (2008): Insular hypocretin transmission regulates nicotine reward. Proc Natl Acad Sci U S A 105:19480-19485.

26. Lawrence AJ, Cowen MS, Yang HJ, Chen F, Oldfield B (2006): The orexin system regulates alcohol-seeking in rats. Br J Pharmacol 148: 752-759.

27. Lawrence AJ (2010): Regulation of alcohol-seeking by orexin (hypocretin) neurons. Brain Res 1314:124-129.

28. Borgland SL, Chang SJ, Bowers MS, Thompson JL, Vittoz N, Floresco SB, et al. (2009): Orexin A/hypocretin-1 selectively promotes motivation for positive reinforcers. J Neurosci 29:11215-11225.

29. España RA, Oleson EB, Locke JL, Brookshire BR, Roberts DC, Jones SR (2010): The hypocretin-orexin system regulates cocaine self-administration via actions on the mesolimbic dopamine system. Eur J Neurosci 31:336-348.

30. Marcus JN, Aschkenasi CJ, Lee CE, Chemelli RM, Saper CB, Yanagisawa M, et al. (2001): Differential expression of orexin receptors 1 and 2 in the rat brain. $J$ Comp Neurol 435:6-25.

31. Cluderay JE, Harrison DC, Hervieu GJ (2002): Protein distribution of the orexin-2 receptor in the rat central nervous system. Regul Pept 104:131-144.

32. Hollander JA, Pham D, Fowler CD, Kenny PJ (2012): Hypocretin-1 receptors regulate the reinforcing and reward-enhancing effects of cocaine: pharmacological and behavioral genetics evidence. Front Behav Neurosci 6:47.

33. Palamarchouk V, Smagin G, Goeders NE (2009): Self-administered and passive cocaine infusions produce different effects on corticosterone concentrations in the medial prefrontal cortex (MPC) of rats. Pharmacol Biochem Behav 94:163168. 
34. Metaxas A, Bailey A, Barbano MF, Galeote L, Maldonado R, Kitchen I (2010): Differential region-specific regulation of $\alpha 4 \beta 2 *$ nAChRs by self-administered and non-contingent nicotine in C57BL/6J mice. Addict Biol 15:464-479.

35. Harris GC, Aston-Jones G (2006): Arousal and reward: a dichotomy in orexin function. Trends Neurosci 29:571-577.

36. Richardson KA, Aston-Jones G (2012): Lateral hypothalamic orexin/hypocretin neurons that project to ventral tegmental area are differentially activated with morphine preference. J Neurosci 32:3809-3817.

37. Morganstern I, Chang GQ, Barson JR, Ye Z, Karatayev O, Leibowitz SF (2010): Differential effects of acute and chronic ethanol exposure on orexin expression in the perifornical lateral hypothalamus. Alcohol Clin Exp Res 34:886-896.

38.Laorden ML, Ferenczi S, Pintér-Kübler B, González-Martín LL, Lasheras MC, Kovács KJ, et al. (2012). Hypothalamic orexin--a neurons are involved in the response of the brain stress system to morphine withdrawal. PLoS One 7:e36871.

39. Tanda G, Pontieri FE, Di Chiara G (1997): Cannabinoid and heroin activation of mesolimbic dopamine transmission by a common mul opioid receptor mechanism. Science 276:2048-2050.

40. Oleson EB, Cheer JF (2012): A brain on cannabinoids: the role of dopamine release in reward seeking. Cold Spring Harb Perspect Med 2(8).

41. Maldonado R, Valverde O, Berrendero F (2006): Involvement of the endocannabinoid system in drug addiction. Trends Neurosci 29:225-232.

42. Mahler SV, Smith RJ, Moorman DE, Sartor GC, Aston-Jones G (2012): Multiple roles for orexin/hypocretin in addiction. Prog Brain Res 198:79-121. 
43. Narita M, Nagumo Y, Hashimoto S, Narita M, Khotib J, Miyatake M, et al. (2006): Direct involvement of orexinergic systems in the activation of the mesolimbic dopamine pathway and related behaviors induced by morphine. $J$ Neurosci 26:398-405.

44. Korotkova TM, Sergeeva OA, Eriksson KS, Haas HL, Brown RE (2003): Excitation of ventral tegmental area dopaminergic and nondopaminergic neurons by orexins/hypocretins. J Neurosci 23:7-11.

45. España RA, Melchior JR, Roberts DC, Jones SR (2011): Hypocretin 1/orexin A in the ventral tegmental area enhances dopamine responses to cocaine and promotes cocaine self-administration. Psychopharmacology (Berl) 214:415-426.

46. Fadel J, Deutch AY (2002): Anatomical substrates of orexin-dopamine interactions: lateral hypothalamic projections to the ventral tegmental area. Neuroscience 111:379-387.

47. Taslimi Z, Haghparast A, Hassanpour-Ezatti M, Safari MS (2011): Chemical stimulation of the lateral hypothalamus induces conditioned place preference in rats: Involvement of $\mathrm{OX} 1$ and $\mathrm{CB} 1$ receptors in the ventral tegmental area. Behav Brain Res 217:41-46.

48. Ward RJ, Pediani JD, Milligan G (2011): Heteromultimerization of cannabinoid $\mathrm{CB}(1)$ receptor and orexin $\mathrm{OX}(1)$ receptor generates a unique complex in which both protomers are regulated by orexin A. J Biol Chem 286:37414-37428.

49. Paxinos G, Franklin KBJ (1997): The mouse brain in stereotaxic coordinates. San Diego Academic Press. 


\section{Figure legends}

Figure 1. Hypocretin receptor-1 blockade reduces the acquisition of operant WIN55,212-2 self-administration. SB334867 (10 mg/kg, ip), TCSOX229 (10 mg/kg, ip) or respective vehicles were administered 30 minutes before each WIN55,212-2 selfadministration training session. (A,D) Percentage of acquisition of WIN55,212-2 selfadministration in (A) vehicle or SB334867 pretreated mice $(n=14-16$ mice per group) and in (D) vehicle or TCSOX229 pretreated mice $(\mathrm{n}=10-13$ mice per group). (B,E) Time course of acquisition of operant WIN55,212-2 self-administration in SB334867, TCSOX229 or vehicle pretreated mice. (C,F) Area under the curve (AUC) values for the number of infusions from training sessions 7 to 12 . (G,I) Time course of acquisition of water-maintained operant conditioning in (G) vehicle or SB334867 pretreated mice $(\mathrm{n}=7$ mice per group) and in (I) vehicle or TCSOX229 pretreated mice $(\mathrm{n}=6-8$ mice per group). (H,J) Breaking-point values achieved in the progressive ratio schedule in SB334867, TCSOX229 or vehicle pretreated mice after water-maintained operant conditioning. Data are expressed as mean \pm SEM. $\star \star p<0.01$ compared with vehicle. VEH, vehicle; SB, SB334867; TCS, TCSOX229; A, active hole; I, inactive hole.

Figure 2. Acute administration of the hypocretin-1 receptor antagonist SB334867 reduces operant WIN55,212-2 self-administration and motivation to obtain the drug. Mice were trained to self-administer WIN55,212-2 during 12 consecutive days and injected with vehicle 30 minutes before each training session. Only those mice achieving acquisition criteria on day 8 were included in the study (see Results section for details). SB334867 (10 mg/kg, ip) or TCSOX229 (10 mg/kg, ip) were administered 30 minutes before the training session on day 9, or 30 minutes before the progressive ratio session. $(\mathbf{A}, \mathbf{B})$ Number of active and inactive responses displayed after acute (A) 
SB334867 $(\mathrm{n}=16)$ or $(\mathbf{B})$ TCSOX229 $(\mathrm{n}=12)$ pretreatment on day 9 , and during the three days before (mean days 6,7,8) and after (mean days 10,11,12) these pharmacological challenges. (C,D) Breaking-point values achieved in the progressive ratio schedule after acute (C) SB334867 or (D) TCSOX229 pretreatment ( $\mathrm{n}=8$-12 mice per group). Data are expressed as mean \pm SEM. $\star \star p<0.01$ compared with vehicle. VEH, vehicle; SB, SB334867; TCS, TCSOX229; A, active hole; I, inactive hole.

Figure 3. Hypocretin receptor-1 gene deletion impairs operant WIN55,212-2 selfadministration. Wild-type and hypocretin receptor-1 knockout mice were trained to self-administer WIN55,212-2 during 12 days. (A) Percentage of acquisition of WIN55,212-2 self-administration in wild-type and knockout mice $(\mathrm{n}=16-18$ mice per group). (B) Time course of acquisition of intravenous WIN55,212-2 self-administration in wild-type and knockout mice. (C) Area under the curve (AUC) values for the number of infusions from training sessions 7 to 12. (D) Breaking-point values achieved in the progressive ratio schedule in wild-type and knockout mice. (E) Time course of acquisition of water-maintained operant conditioning in wild-type and knockout mice (n =7-8 mice per group). (F) Breaking-point values achieved in progressive ratio schedule in wild-type and knockout mice after water-maintained operant conditioning. Data are expressed as mean \pm SEM. $\star \mathrm{p}<0.05, \star \star \mathrm{p}<0.01$ compared with wild-type mice. WT, wild type mice; KO, Hcrtr-1 knockout mice.

Figure 4. Contingent, but not non-contingent WIN55,212-2 self-administration activates hypocretin neurons in the lateral hypothalamus. Double label immunofluorescence of $\mathrm{Fos} B / \Delta$ fosB with hypocretin-1 was performed in the lateral (LH) as well as dorsomedial and perifornical (DMH/PFA) hypothalamus of mice which 
had underwent a contingent/non-contingent WIN55,212-2 self-administration paradigm during 12 days. $(\mathbf{A}, \mathbf{B}, \mathbf{C})$ Operant responding on the active and inactive holes in mice receiving (A) passive saline infusions (saline-yoked mice), (B) passive intravenous WIN55,212-2 infusions (WIN-yoked mice), and (C) contingent intravenous WIN55,212-2 infusions (WIN-contingent) $(\mathrm{n}=7$ mice per group). (D,E) Percentage of FosB/ $\Delta$ FosB positive hypocretin-1 expressing neurons after contingent/non-contingent WIN55,212-2 self-administration in the (D) LH and the (E) DMH/PFA. (F) Representative images of sections of the LH obtained via fluorescence microscopy after direct double labeling combining rabbit polyclonal antiserum to $F o s B / \Delta F o s B$ with mouse monoclonal antibody to hypocretin-1. Arrowheads indicate FosB/ $\Delta$ FosB positive hypocretin-1 expressing neurons. The scale bar represents $100 \mu \mathrm{m}$ in upper images and $25 \mu \mathrm{m}$ in lower images. (G) Schematic anatomic representation of LH subdivisions (bregma $-1.70 \mathrm{~mm}$ ) adapted from Paxinos and Franklin's stereotaxic atlas (49). Labeled areas delineate regions where $\mathrm{Fos} B / \Delta$ FosB expression was examined. (H,I) Operant responding on the active and inactive holes in mice receiving $(\mathbf{H})$ passive (water-yoked mice) or (I) contingent (water-contingent mice) water delivery ( $\mathrm{n}=7$ mice per group). (J,K) Percentage of FosB/ $\Delta$ FosB positive hypocretin-1 expressing neurons after contingent/non-contingent water-maintained conditioning in the $(\mathbf{J})$ LH and the $(\mathbf{K})$

DMH/PFA. Data are expressed as mean \pm S.E.M. $\star \mathrm{p}<0.05$ comparison between groups.

Figure 5. THC-induced enhancement in dopamine extracellular levels in the nucleus accumbens is blocked in hypocretin receptor-1 knockout mice. (A) Representative coronal section of a mouse brain stained with cresyl violet showing a microdialysis probe placement. (B) Location of the probes for each of the mice included 
in the study (between AP, bregma +1.10 and $+1.54 \mathrm{~mm}$ ). (C) Time course of basal and stimulated levels of dopamine (percentage from baseline) in the nucleus accumbens. Dialysates were collected 80 minutes before and 160 minutes after a challenge injection of THC $(0.3 \mathrm{mg} / \mathrm{kg}$, ip; arrow at time 0$)$ or vehicle, in wild-type and hypocretin receptor-1 knockout mice ( $\mathrm{n}=10-14$ mice per group). (D) Area under the curve (AUC) values for levels after THC or vehicle injection (from 0 to 160 minutes) for the different groups of mice. Data are expressed as mean \pm SEM. $\star \star p<0.01$ compared with wildtype mice. \#\#p<0.01 compared with vehicle. WT, wild-type mice; KO, hypocretin receptor-1 knockout mice; VEH, vehicle. 